\title{
'Best care' is not good enough
}

New research shows that only $9 \%$ of men with localized prostate cancer in the USA received 'best care' according to nationally endorsed care measures. Moreover, according to the study published in The Journal of Urology, these measures do not seem to be meaningful in terms of outcomes.

\section{Further research is needed} to identify better measures that are more tightly linked to outcomes... 77

In their retrospective cohort study, Schroeck et al. assessed data from 38,055 men with localized prostate cancer who had been treated between 2004 and 2010 . The researchers determined whether each patient had received best care according to a set of five nationally endorsed measures to assess quality of care. Associations between best care and treatment-related morbidity and cancer control were then assessed. Patients were grouped according to the primary treatment they received: prostatectomy; radiotherapy only; or radiotherapy with concurrent androgen-deprivation therapy.

Only 3,412 men were found to have received best care. These men were more likely to be older, to have immediate-risk disease and be of higher socioeconomic status than men who did not receive best care. "When examining the associations between receipt of best care and outcomes, our findings were mixed," explains first author Florian Schroeck.

For instance, best care was associated with a decreased risk of gastrointestinal complications among patients undergoing radiotherapy but not concurrent androgen-deprivation therapy, whilst men who underwent prostatectomy and received best care were more likely to receive treatment for sexual morbidity. Crucially, for most patient groups, best care was not associated with improved outcomes in terms of cancer control and treatment-related morbidity. Whether or not they received best care, most men had a similar likelihood of therapy for secondary cancer or to undergo procedures for urinary, sexual or gastrointestinal adverse effects 5 years after primary treatment.

The study authors note that the fact that only a minority of patients received best care underlines how difficult it can be to consistently adhere to recommended processes of care. "Further research is needed to identify better measures that are more tightly linked to outcomes or are clearly meaningful to patients," concludes Schroeck. "Development of such measures will hopefully be facilitated by new national registries."

Katrina Ray

Original article Schroeck, F. R. et al. Receipt of "best care" according to current quality of care measures and outcomes among men with prostate cancer. J. Urol. doi:10.1016/j.juro.2014.08.001 